\title{
Entropic Bounds on Information Backflow
}

\author{
Nina Megier®, ${ }^{*}$ Andrea Smirne $\odot$, and Bassano Vacchini® \\ Dipartimento di Fisica “Aldo Pontremoli," Università degli Studi di Milano, via Celoria 16, 20133 Milan, Italy \\ and Istituto Nazionale di Fisica Nucleare, Sezione di Milano, via Celoria 16, 20133 Milan, Italy
}

(Received 24 December 2020; accepted 7 June 2021; published 14 July 2021)

\begin{abstract}
In the dynamics of open quantum systems, the backflow of information to the reduced system under study has been suggested as the actual physical mechanism inducing memory and thus leading to nonMarkovian quantum dynamics. To this aim, the trace-distance revivals between distinct evolved system states have been shown to be subordinated to the establishment of system-environment correlations or changes in the environmental state. We show that this interpretation can be substantiated also for a class of entropic quantifiers. We exploit a suitably regularized version of Umegaki's quantum relative entropy, known as telescopic relative entropy, that is tightly connected to the quantum Jensen-Shannon divergence. In particular, we derive general upper bounds on the telescopic relative entropy revivals conditioned and determined by the formation of correlations and changes in the environment. We illustrate our findings by means of examples, considering in particular the Jaynes-Cummings model and a phase covariant dynamics.
\end{abstract}

DOI: 10.1103/PhysRevLett.127.030401

In recent years a wide variety of different definitions of quantum non-Markovianity have been proposed, all of them aimed to reveal the occurrence of memory effects in quantum evolutions [1-4]. The most widespread are the ones based on the divisibility property of the dynamical map [5-8], the monotonicity of the trace distance (TD) between two distinct reduced states [8-10], the change of the volume of accessible reduced states [11], and the process tensor formalism [12-14]. Furthemore, entropic quantities have also been used to detect non-Markovianity, see [15-17]. The interest toward non-Markovian quantum dynamics has not only theoretical motivations: nonMarkovianity has proven to be beneficial, among others, in quantum control [18] and teleportation tasks [19]. As the different definitions of quantum non-Markovianity are in general not equivalent, it is all the more important to find their corresponding physical interpretations. In the approach based on TD, its increase in time signifies a backflow of information to the open system, resulting in an enhanced reduced-state distinguishability and representing the distinctive trait of memory effects in the dynamics. The revivals of distinguishability are related to the establishment of system-environment correlations and changes in the environmental state depending on the initial system state [20-24], though the precise assessment of their role is still under vivid debate [25-30].

The proof of the connection of the distinguishability revivals with correlations and environmental state changes as formulated via the TD essentially relies on the triangle inequality, so that it might be natural to think that it only holds when distance quantifiers are used. Here we show that, to the contrary, such a connection can be maintained also when considering entropic quantifiers. Our analysis extends and strengthens the viewpoint that quantum nonMarkovianity can be understood in terms of a backflow of information, which induces an increase of the distinguishability among open-system states and is microscopically motivated by the generation of correlations and changes in the environmental state due to the system-environment interaction. More specifically, we prove an upper bound for the revivals in time of a whole class of entropic distinguishability quantifiers, namely the telescopic relative entropy (TRE), also called quantum skew divergence, introduced in $[31,32]$ and providing regularized versions of the quantum relative entropy (QRE). Remarkably, our bound links quantitatively the distinguishability revivals with the establishment of correlations between the system and the environment due to their mutual interaction, as well as to the modification of the state of the environment. We also focus on a special case of the symmetrized version of TRE, which coincides with the quantum Jensen-Shannon divergence (QJSD), a widely used distinguishability measure [33-40]. We show that the upper bound to the distinguishability revivals in this case significantly simplifies and becomes tighter. Finally, we showcase our findings on several examples, including the paradigmatic JaynesCummings model and a phase covariant dynamics of a qubit, which allows us to compare explicitly the behaviors of the different quantifiers of distinguishability involved in our analysis.

Information backflow and non-Markovianity.-The TD is defined as $\mathrm{D}(\varrho, \sigma)=1 / 2 \operatorname{Tr}|\varrho-\sigma|$, and provides a natural distance on the space of statistical operators [41]. Its crucial feature allowing to define and identify memory 
effects is the contractivity under the action of a (completely) positive trace preserving ((C)PT) map $\Phi$; namely, TD obeys the so-called data processing inequality

$$
\mathrm{D}(\Phi[\varrho], \Phi[\sigma]) \leq \mathrm{D}(\varrho, \sigma)
$$

for any pair of states $Q, \sigma$ [42]. Importantly, this property brings with itself invariance under unitary maps and with respect to the tensor product, i.e., $\mathrm{D}(\varrho, \sigma)=\mathrm{D}(\varrho \otimes \tau, \sigma \otimes \tau)$ for any state $\tau$, as can be readily seen using CPT of both the partial trace and the map $\varrho \mapsto \varrho \otimes \tau$. In particular, TD is a proper quantum $f$ divergence, which allows us to use it to quantify the distinguishability between quantum states [44]. Finally, we consider two further important properties of the TD, which are an immediate consequence of its being a distance in the mathematical sense. The first is the validity of the triangle inequality, which can be expressed as

$$
\begin{aligned}
& \mathrm{D}(\varrho, \sigma)-\mathrm{D}(\varrho, \tau) \leq \mathrm{D}(\sigma, \tau), \\
& \mathrm{D}(\varrho, \sigma)-\mathrm{D}(\eta, \sigma) \leq \mathrm{D}(\varrho, \eta),
\end{aligned}
$$

for arbitrary states $\varrho, \sigma, \tau$, and $\eta$. The second is positivity and boundedness according to

$$
0 \leq \mathrm{D}(\varrho, \sigma) \leq 1
$$

with the value 0 if and only if $Q=\sigma$, and 1 if and only if their supports are orthogonal. Thanks to the properties given by Eqs. (1)-(4) one can derive an upper bound for the difference of the TD between the open system states $\varrho_{S}, \sigma_{S}$, at different times $t, s: t \geq s$. These states result from two distinct initial conditions given by factorized states with the same environmental marginal, $\varrho(0)=\varrho_{S}(0) \otimes \varrho_{E}(0)$, $\sigma(0)=\sigma_{S}(0) \otimes \sigma_{E}(0)$, with $\sigma_{E}(0)=\varrho_{E}(0)$, so as to ensure the existence of a reduced dynamics [20,24,26]. The bound reads

$$
\begin{aligned}
& \mathrm{D}\left(\varrho_{S}(t), \sigma_{S}(t)\right)-\mathrm{D}\left(\varrho_{S}(s), \sigma_{S}(s)\right) \leq \mathrm{D}\left(\varrho_{E}(s), \sigma_{E}(s)\right) \\
& \quad+\mathrm{D}\left(\varrho(s), \varrho_{S}(s) \otimes \varrho_{E}(s)\right)+\mathrm{D}\left(\sigma(s), \sigma_{S}(s) \otimes \sigma_{E}(s)\right) .
\end{aligned}
$$

The interpretation of this inequality is central to our analysis and relies on the TD as quantifier of distinguishability among quantum states, so that it can be used to identify the different contributions to the exchange of information between the open system and the environment. The lhs of Eq. (5) quantifies the amount of information gained or lost by the reduced system from time $s$ to time $t$. Note that the quantity is negative for $s=0$, as the information is initially fully contained within the open quantum system. On the other hand, the terms at the rhs of Eq. (5) quantify the information that is outside the open system at time $s$, i.e., that can be accessed only with measurements involving also environmental degrees of freedom. Such information can be encoded in the correlations between the open system and the environment or in the environmental state. Indeed, although the environmental states are initially the same, they will generally differ at later times, due to the initial difference in the reduced states $\varrho_{S}(0)$ and $\sigma_{S}(0)$. Any backflow of information towards the open system in the interval of time from $s$ to $t$ will thus result in a positive value on the lhs of Eq. (5). As the global system is closed, this additional information at time $t$ must have an environmental origin, i.e., at time $s$ it was contained in the environmental state or in the correlations between system and environment, which is precisely what is quantified by Eq. (5).

We stress that any distance contractive under (C)PT maps, e.g., the Bures distance considered in [24,45], would lead to an inequality analogous to the one in Eq. (5) and would then allow for the same physical interpretation. However, till now no entropic quantifier could be used for this purpose, as no entropic distance measure was known, though it was conjectured long ago that the square root of the QJSD is actually a metric $[46,47]$. This was recently shown $[48,49]$, more than ten years after the proof for the classical Jensen-Shannon divergence [50]. Here, we show that there is a whole class of entropic quantities that are in general not distance measures but also allow for the interpretation above; such a class includes QJSD as a special case. Thus, we substantiate the fact that the actual physical mechanism behind the occurrence of memory effects in quantum dynamics is the establishment of correlations or changes in the environmental states upper bounding the revivals in local distinguishability.

Telescopic relative entropy.-Relative entropy is a fundamental quantity in statistical mechanics and information theory, both at the classical and quantum level [51-53]. It can be interpreted as a quantifier of the error rate in discriminating two probability distributions in the limit of many measurement repetitions and it also plays a distinguished role in quantum thermodynamics and its foundations, especially in analyzing the formulation of the second law of thermodynamics in the quantum regime [54-57]. The expression of the QRE first introduced by Umegaki [58] reads $S(\varrho, \sigma)=\operatorname{Tr}(\varrho \log \varrho-\varrho \log \sigma)$. As well known the QRE, while being the most relevant quantum $f$ divergence distinguishing quantum states [44], is not bounded and can diverge also in finite dimension. To cure this difficulty, regularized versions have been proposed $[31,33,59]$. We will show that the TRE introduced in [31,32] obeys an analog of the key inequalities Eqs. (1)-(5). For a special choice of the telescopic parameter introduced below, the symmetrized version of TRE reduces to the QJSD, and a simplified upper bound for its square root follows. With this, also for this class of entropic quantifiers an interpretation of the 
distinguishability revivals in terms of information backflow is given. The TRE is defined as

$$
\mathrm{S}_{\mu}(\varrho, \sigma)=\log (1 / \mu)^{-1} S(\varrho, \mu \varrho+(1-\mu) \sigma)
$$

and is actually independent of the logarithm basis used in the definition. The telescopic parameter $\mu \in(0,1)$ gives the amount of mixing between the two states $Q$ and $\sigma$. For $\mu=1 / 2$ the symmetrized TRE $\mathrm{J}(\varrho, \sigma)=1 / 2\left[S_{1 / 2}(\varrho, \sigma)+\right.$ $\left.S_{1 / 2}(\sigma, \varrho)\right]$ equals the QJSD [33]:

$$
\mathrm{J}(\varrho, \sigma)=\frac{1}{2}\left[S\left(\varrho, \frac{\varrho+\sigma}{2}\right)+S\left(\sigma, \frac{\varrho+\sigma}{2}\right)\right] .
$$

The main property of the TRE, which distinguishes it from the standard QRE, is its boundedness. In particular, the prefactor is chosen so that $0 \leq \mathrm{S}_{\mu}(\varrho, \sigma) \leq 1$, assuming the extreme values if and only if the states are identical or have orthogonal support [31,32]. Moreover, TRE inherits from the QRE the joint convexity and the contractivity under (C) PT maps [60]

$$
\mathrm{S}_{\mu}(\Phi[\varrho], \Phi[\sigma]) \leq \mathrm{S}_{\mu}(\varrho, \sigma) .
$$

Neither QRE nor TRE are distances as they do not satisfy the triangle inequality and are not symmetric in their arguments. However, it can be shown that TRE obeys the following inequalities [32,61], similar in spirit to Eq. (2) and Eq. (3):

$$
\begin{array}{r}
\mathrm{S}_{\mu}(\varrho, \sigma)-\mathrm{S}_{\mu}(\varrho, \tau) \leq 1-\mathrm{S}_{\mu}(1, \mathrm{D}(\sigma, \tau)) \\
\mathrm{S}_{\mu}(\varrho, \sigma)-\mathrm{S}_{\mu}(\eta, \sigma) \leq \mathrm{D}(\varrho, \eta)-\mathrm{S}_{\mu}(\mathrm{D}(\varrho, \eta), 1)
\end{array}
$$

where we have generalized the definition of TRE to act on non-negative scalars. The TRE can be bounded from below and above by functions of the TD

$$
2(1-\mu)^{2} \log (1 / \mu)^{-1} \mathrm{D}^{2}(\varrho, \sigma) \leq \mathrm{S}_{\mu}(\varrho, \sigma) \leq \mathrm{D}(\varrho, \sigma),
$$

where the lower bound follows from the Pinsker inequality for the QRE [62]. Exploiting these properties, we derive, as detailed in [63], the following inequality for the change in TRE:

$$
\begin{array}{r}
\mathrm{S}_{\mu}\left(\varrho_{S}(t), \sigma_{S}(t)\right)-\mathrm{S}_{\mu}\left(\varrho_{S}(s), \sigma_{S}(s)\right) \leq \kappa_{\mu}\left\{\mathrm{S}_{\mu}^{1 / 4}\left(\varrho_{E}(s), \sigma_{E}(s)\right)\right. \\
\left.+\mathrm{S}_{\mu}^{1 / 4}\left(\varrho(s), \varrho_{S}(s) \otimes \varrho_{E}(s)\right)+\mathrm{S}_{\mu}^{1 / 4}\left(\sigma(s), \sigma_{S}(s) \otimes \sigma_{E}(s)\right)\right\}
\end{array}
$$

with $\kappa_{\mu}=\left[2 \mu^{2} \log ^{3}(1 / \mu)\right]^{-1 / 4}$. While boundedness of the TRE allows us to introduce a well-defined nonMarkovianity measure as for the TD [3], this bound permits a full-fledged interpretation of TRE as a quantifier of information backflow. Note that the prefactor $\kappa_{\mu}$ as a function of $\mu$ takes a global minimum $\left(4 e^{3} / 27\right)^{1 / 4} \approx$ 1.31 at $\mu=e^{-3 / 2}$, which we will take as a reference value of the telescopic parameter in the figures. For $\mu=1 / 2$, the symmetrized TRE corresponds to the QJSD J $(\varrho, \sigma)$ of Eq. (7), whose square root is a distance. With this and the contractivity under CPT maps one has

$$
\begin{aligned}
& \sqrt{\mathrm{J}\left(\varrho_{S}(t), \sigma_{S}(t)\right)}-\sqrt{\mathrm{J}\left(\varrho_{S}(s), \sigma_{S}(s)\right)} \leq \sqrt{\mathrm{J}\left(\varrho_{E}(s), \sigma_{E}(s)\right)} \\
& +\sqrt{\mathrm{J}\left(\varrho(s), \varrho_{S}(s) \otimes \varrho_{E}(s)\right)}+\sqrt{\mathrm{J}\left(\sigma(s), \sigma_{S}(s) \otimes \sigma_{E}(s)\right)} .
\end{aligned}
$$

Note, that in the case of TD one can show that states maximizing the backflow of information are always orthogonal and lie on the boundary of the space of states [65], while a comparable characterization for TRE is still missing.

Examples.-We now showcase our findings by examples. Let us consider first the Jaynes-Cummings model, describing the interaction of a qubit with a single bosonic field mode

$H=\omega_{S} \sigma_{z} \otimes \mathbb{I}+g\left(\sigma_{+} \otimes b+\sigma_{-} \otimes b^{\dagger}\right)+\omega_{E} \mathbb{I} \otimes b^{\dagger} b$,

where we introduced the raising and lowering operators $\sigma_{ \pm}=\sigma_{x} \pm i \sigma_{y}$ expressed in terms of the Pauli matrices, while $b, b^{\dagger}$ are bosonic annihilation and creation operators, respectively. This model can be solved exactly $[66,67]$, thus allowing for a comparison of the TD and TRE quantities occurring in Eqs. (5), (12), and (13). In Fig. 1 we report in each panel the lhs and the three contributions at the rhs of the bounds for the TD, the TRE, and the square root of the QJSD, which we denote as QJSD $^{1 / 2}$, respectively. We see that the qualitative behavior of these quantifiers of the distinguishability of quantum states is similar, with respect to both the information contained within the open system and the one outside it, namely, the system-environment correlations and environmental states. The quantities referred to the QJSD ${ }^{1 / 2}$ in particular follow very tightly the behavior of the corresponding TD quantities. The TRE is always smaller than the corresponding TD, which is a general feature for all telescopic parameters $\mu$, see Eq. (11). From this, however, one cannot conclude that the terms appearing in Eq. (12) are always smaller than the corresponding ones in Eq. (5), as can be seen in Fig. 2. Actually, the upper bounds in terms of the entropic quantities are almost always less tight for this model than the corresponding TD one. As all three bounds are for most of the time above one, their applicability for estimation of the $\mathrm{lhs}$, which is never larger than one, is limited. Nonetheless, their existence guarantees the direct relation to information backflow, as the revival of local distinguishability unambiguously originates from establishment of correlations or 

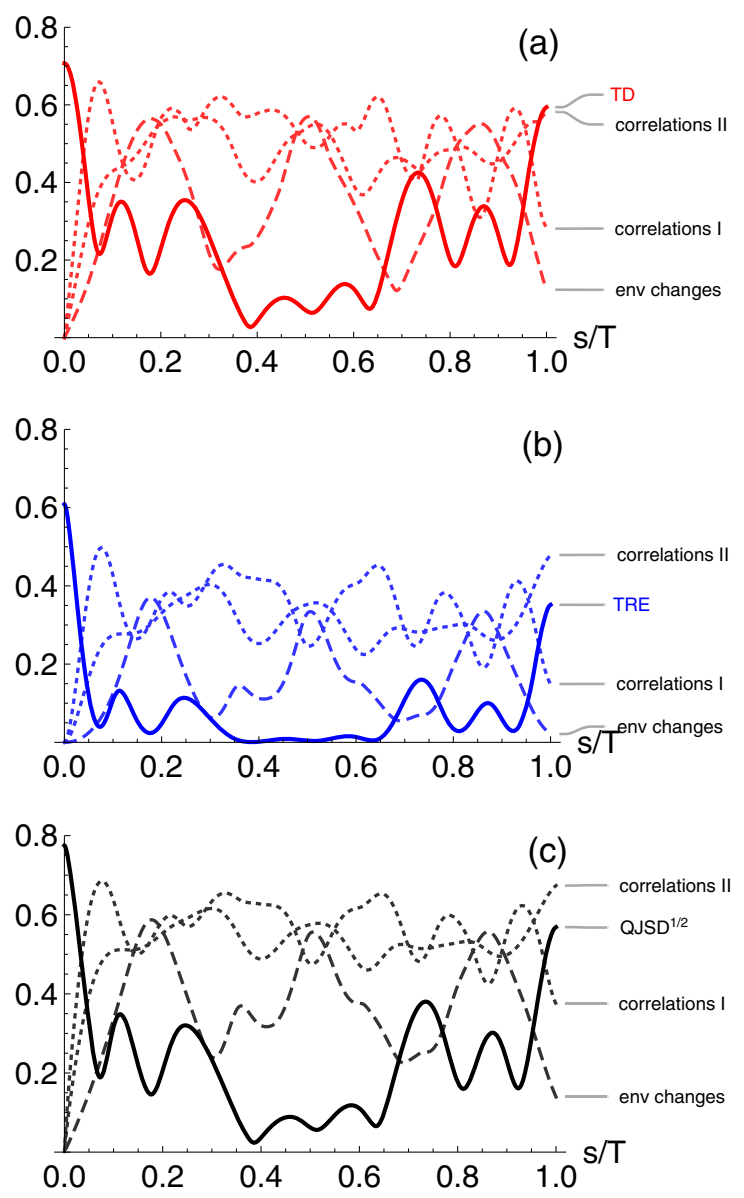

FIG. 1. Different contributions to the bounds for the considered distinguishability quantifiers for the Jaynes-Cummings model: (a) the solid line corresponds to the TD as a function of the rescaled time; the dashed line is the first contribution at the rhs of Eq. (5) corresponding to different changes in the environmental states, while the two dotted lines correspond to system-environment correlations, respectively second (I) and third (II) contributions at the rhs of Eq. (5); (b) and (c) provide the corresponding quantities relative to TRE for $\mu=e^{-3 / 2}$ and QJSD ${ }^{1 / 2}$. The initial states of the qubit are given by the excited state $|1\rangle$ and a symmetric superposition of excited and ground state, $1 / \sqrt{2}(|1\rangle+|0\rangle)$, while the environment starts in a thermal state with $\beta \omega_{E}=1$, where $k_{B} \beta$ denotes the inverse temperature of the bath. We set the time $T$ equal to 8.9 in inverse units of the coupling strength $g$, while the detuning frequency $\omega_{S}-\omega_{E}$ is equal to 0.5 in units of $g$.

changes in the environmental states, thus acting as precursors of non-Markovianity [24]. In particular, one can consider physical models for which only the system-environment correlations are responsible for information backflow at all times and the upper bounds in Eqs. (5), (12), and (13) directly quantify them. This is the case for the spin-star dephasing model [68], as detailed in Ref. [63]. On the other hand, considering a simple two-qubit model where the global correlations vanish at isolated instants of time, it is possible to show that the occurrence of the roots in Eq. (12)

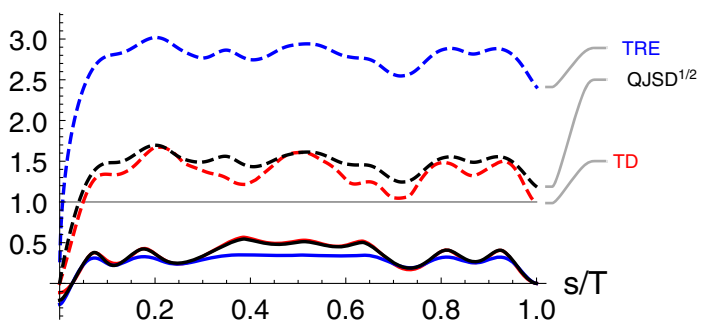

FIG. 2. Revivals of distance and entropic distinguishability quantifiers versus their bounds in terms of correlations and environmental changes for the Jaynes-Cummings model (all parameters as in Fig. 1): TD (red), TRE (blue), and QJSD ${ }^{1 / 2}$ (black). Solid and dashed lines correspond, respectively, to the lhs and rhs of Eqs. (5), (12), and (13). The straight line at 1 corresponds to the maximal possible value of the revivals. As in Fig. 1 the value of $t$ at the lhs of the bounds is set to $T=8.9$ in inverse units of the coupling strength $g$, corresponding to a local maximum of the distinguishability. The very close behavior of $\mathrm{TD}$ and $\mathrm{QJSD}^{1 / 2}$ clearly appears.

and Eq. (13) makes the TRE and QJSD ${ }^{1 / 4}$ more sensitive than the TD to the changes in the system-environment correlations in points in time at which they vanish [63].

In the previous example we have observed a similar behavior in the evolutions of the TD and the TRE. This is, however, not always the case, as we will now show via a qubit phase covariant dynamics (for a discussion of this class of quantum evolutions and its relevance for metrological tasks see [69]). Consider the dynamics [70,71]

$\varrho(t)=\frac{1}{2}\left[1+r(t) \sigma_{z}+\eta_{\perp}(t)\left(v_{x} \sigma_{x}+v_{y} \sigma_{y}\right)+\eta_{\|}(t) v_{z} \sigma_{z}\right]$,

where $v_{i}=\operatorname{Tr}\left[\sigma_{i} \varrho(0)\right]$, causing the Bloch ball to shrink into an ellipsoid with axes of length $\left|\eta_{\perp}(t)\right|$ in the $x$ and $y$ directions and $\left|\eta_{\|}(t)\right|$ in the $z$ direction, and to shift along the $z$ axis by $r(t)$. In Fig. 3 we consider the evolution of the entropic distinguishability measures and TD for a relaxation of the open system to the maximally mixed state, with long-lasting oscillations of the state translation, i.e., of the nonunital component of the dynamics. As both the functions $\eta_{\|}(t)$ and $\eta_{\perp}(t)$ are monotonically decreasing the same is true for the TD between two arbitrary initial states [72]. On the other hand, the TRE and $\mathrm{QJSD}^{1 / 2}$ are characterized by revivals. Thus, we conclude that while the TD is not sensitive to the non-Markovianity due to the nonunital part of the dynamics since it is a function of the difference between states [73], the entropic distinguishability measures are able to detect the backflow of information to the open system originating from nonunital contributions.

Conclusions.-We have shown that entropic quantities can be used to consistently define the exchange of information between an open quantum system and its 


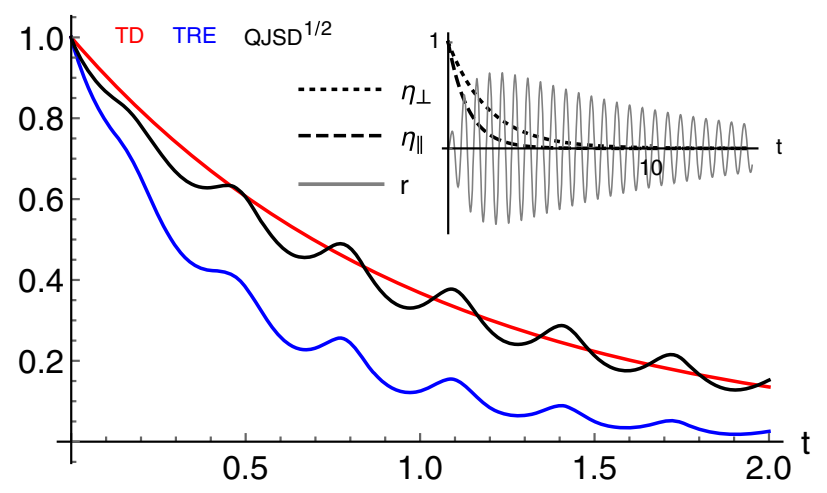

FIG. 3. TD (red), TRE (blue), and QJSD ${ }^{1 / 2}$ (black) between the reduced states obtained starting from the excited and ground state as functions of time $t$ in inverse units of the longitudinal damping rate. The system obeys the phase covariant dynamics Eq. (15) with the functions $\eta_{\perp}(t)$ (dotted), $\eta_{\|}(t)$ (dashed) and $r(t)$ (solid) as in the inset.

environment. By focusing on a class of regularized versions of the quantum relative entropy, named telescopic relative entropy, we derived an upper bound to the variation of the reduced state distinguishability in terms of the information lying outside the open system, encoded in the systemenvironment correlations and the environmental states. Besides strengthening the interpretation of nonMarkovianity as backflow of information, our results also clarify which are the key mathematical properties behind this picture. We have further put into evidence the distinguished role of the Jensen-Shannon divergence, providing a special case of telescopic relative entropy, that appears as the most natural entropic quantifier of non-Markovianity. In addition, we have investigated in examples the relation between telescopic relative entropy and trace distance, showing in particular that, contrary to the latter, the former is able to detect the non-Markovianity originating from the nonunital part of the dynamics. In future studies, it will be important to understand to what extent the use of entropic quantities to characterize non-Markovian open system dynamics can be further justified, developing a measure of non-Markovianity by the identification of the pair of states maximizing the backflow of information $[9,65]$.

N.M. acknowledges funding by the Alexander von Humboldt Foundation in form of a Feodor-Lynen Fellowship. All authors acknowledge support from the UniMi Transition Grant H2020.

*nina.megier@mi.infn.it, she/her/hers

[1] H.-P. Breuer, J. Phys. B 45, 154001 (2012).

[2] Á. Rivas, S. F. Huelga, and M. B. Plenio, Rep. Prog. Phys. 77, 094001 (2014).

[3] H.-P. Breuer, E.-M. Laine, J. Piilo, and B. Vacchini, Rev. Mod. Phys. 88, 021002 (2016).
[4] L. Li, M. J. W. Hall, and H. M. Wiseman, Phys. Rep. 759, 1 (2018).

[5] M. M. Wolf, J. Eisert, T. S. Cubitt, and J. I. Cirac, Phys. Rev. Lett. 101, 150402 (2008).

[6] A. Rivas, S. F. Huelga, and M. B. Plenio, Phys. Rev. Lett. 105, 050403 (2010).

[7] M. J. W. Hall, J. D. Cresser, L. Li, and E. Andersson, Phys. Rev. A 89, 042120 (2014).

[8] S. Wißmann, H.-P. Breuer, and B. Vacchini, Phys. Rev. A 92, 042108 (2015).

[9] H.-P. Breuer, E.-M. Laine, and J. Piilo, Phys. Rev. Lett. 103, 210401 (2009).

[10] D. Chruściński, A. Kossakowski, and A. Rivas, Phys. Rev. A 83, 052128 (2011).

[11] S. Lorenzo, F. Plastina, and M. Paternostro, Phys. Rev. A 88, 020102(R) (2013).

[12] F. A. Pollock, C. Rodríguez-Rosario, T. Frauenheim, M. Paternostro, and K. Modi, Phys. Rev. A 97, 012127 (2018).

[13] S. Milz, M. S. Kim, F. A. Pollock, and K. Modi, Phys. Rev. Lett. 123, 040401 (2019).

[14] Y.-Y. Hsieh, Z.-Y. Su, and H.-S. Goan, Phys. Rev. A 100, 012120 (2019).

[15] F. F. Fanchini, G. Karpat, B. Cakmak, L. K. Castelano, G. H. Aguilar, O. J. Farias, S. P. Walborn, P. H. Souto Ribeiro, and M. C. de Oliveira, Phys. Rev. Lett. 112, 210402 (2014).

[16] S. Haseli, G. Karpat, S. Salimi, A. S. Khorashad, F. F. Fanchini, B. Cakmak, G. H. Aguilar, S. P. Walborn, and P. H. Souto Ribeiro, Phys. Rev. A 90, 052118 (2014).

[17] J. Kołodyński, S. Rana, and A. Streltsov, Phys. Rev. A 101, 020303(R) (2020).

[18] D. M. Reich, N. Katz, and C. P. Koch, Sci. Rep. 5, 12430 (2015).

[19] E.-M. Laine, H.-P. Breuer, and J. Piilo, Sci. Rep. 4, 4620 (2014).

[20] E.-M. Laine, J. Piilo, and H.-P. Breuer, Europhys. Lett. 92, 60010 (2010).

[21] L. Mazzola, C. A. Rodríguez-Rosario, K. Modi, and M. Paternostro, Phys. Rev. A 86, 010102(R) (2012).

[22] A. Smirne, L. Mazzola, M. Paternostro, and B. Vacchini, Phys. Rev. A 87, 052129 (2013).

[23] S. Cialdi, A. Smirne, M. G. A. Paris, S. Olivares, and B. Vacchini, Phys. Rev. A 90, 050301(R) (2014).

[24] S. Campbell, M. Popovic, D. Tamascelli, and B. Vacchini, New J. Phys. 21, 053036 (2019).

[25] N. Megier, D. Chruściński, J. Piilo, and W. T. Strunz, Sci. Rep. 7, 6379 (2017).

[26] H.-P. Breuer, G. Amato, and B. Vacchini, New J. Phys. 20, 043007 (2018).

[27] A. A. Budini, Phys. Rev. A 97, 052133 (2018).

[28] D. D. Santis and M. Johansson, New J. Phys. 22, 093034 (2020).

[29] D. De Santis, M. Johansson, B. Bylicka, N. K. Bernardes, and A. Acín, Phys. Rev. A 102, 012214 (2020).

[30] M. Banacki, M. Marciniak, K. Horodecki, and P. Horodecki, arXiv:2008.12638.

[31] K. M. R. Audenaert, in Theory of Quantum Computation, Communication, and Cryptography, edited by D. Bacon, M. Martin-Delgado, and M. Roetteler (Springer, Berlin, 2014), pp. 39-52.

[32] K. M. R. Audenaert, J. Math. Phys. (N.Y.) 55, 112202 (2014). 
[33] A. P. Majtey, P. W. Lamberti, and D. P. Prato, Phys. Rev. A 72, 052310 (2005).

[34] W. Roga, M. Fannes, and K. Życzkowski, Phys. Rev. Lett. 105, 040505 (2010).

[35] J. Dajka, J. Łuczka, and P. Hänggi, Phys. Rev. A 84, 032120 (2011).

[36] S. Wißmann, B. Leggio, and H.-P. Breuer, Phys. Rev. A 88, 022108 (2013).

[37] L. Rossi, A. Torsello, E. R. Hancock, and R. C. Wilson, Phys. Rev. E 88, 032806 (2013).

[38] M. De Domenico, V. Nicosia, A. Arenas, and V. Latora, Nat. Commun. 6, 6864 (2015).

[39] C. Radhakrishnan, M. Parthasarathy, S. Jambulingam, and T. Byrnes, Phys. Rev. Lett. 116, 150504 (2016).

[40] A. Slaoui, A. Salah, and M. Daoud, Physica (Amsterdam) 558A, 124946 (2020).

[41] A.S. Holevo, Statistical Structure of Quantum Theory, Lecture Notes in Physics Vol. 67 (Springer, Berlin, 2001).

[42] The data processing inequality is usually stated for $C P$ maps. For trace distance and quantum relative entropy positivity of the map is sufficient [43].

[43] S. Khatri and M. M. Wilde, arXiv:2011.04672.

[44] F. Hiai and M. Mosonyi, Rev. Math. Phys. 29, 1750023 (2017).

[45] R. Vasile, S. Maniscalco, M. G. A. Paris, H.-P. Breuer, and J. Piilo, Phys. Rev. A 84, 052118 (2011).

[46] P. W. Lamberti, A. P. Majtey, A. Borras, M. Casas, and A. Plastino, Phys. Rev. A 77, 052311 (2008).

[47] J. Briët and P. Harremoës, Phys. Rev. A 79, 052311 (2009).

[48] D. Virosztek, Adv. Math. 380, 107595 (2021).

[49] S. Sra, Linear Algebra Appl. 616, 125 (2021).

[50] D. M. Endres and J. E. Schindelin, IEEE Trans. Inf. Theory 49, 1858 (2003).

[51] A. Wehrl, Rev. Mod. Phys. 50, 221 (1978).

[52] B. Schumacher and M. Westmoreland, arXiv:quant-ph/ 0004045.

[53] V. Vedral, Rev. Mod. Phys. 74, 197 (2002).

[54] T. Sagawa, in Lectures on Quantum Computing, Thermodynamics and Statistical Physics, edited by M. Nakahara and S. Tanaka (World Scientific, Singapore, 2012), pp. 125-190.
[55] M. Esposito, K. Lindenberg, and C. V. den Broeck, New J. Phys. 12, 013013 (2010).

[56] K. Ptaszynski and M. Esposito, Phys. Rev. Lett. 123, 200603 (2019).

[57] S. Floerchinger and T. Haas, Phys. Rev. E 102, 052117 (2020).

[58] H. Umegaki, Kodai Math. Sem. Rep. 14, 59 (1962).

[59] K. Lendi, F. Farhadmotamed, and A. J. van Wonderen, J. Stat. Phys. 92, 1115 (1998).

[60] A. Müller-Hermes and D. Reeb, Ann. Inst. Henri Poincaré 18, 1777 (2017).

[61] K. M. R. Audenaert, arXiv:1102.3041.

[62] A. A. Fedotov, P. Harremoes, and F. Topsoe, IEEE Inf. Theory 49, 1491 (2003).

[63] See Supplemental Material at http://link.aps.org/ supplemental/10.1103/PhysRevLett.127.030401 for details, which includes Ref. [64].

[64] F. Topsoe, in Inequality Theory and Applications, edited by Y. J. Cho, J. K. Kim, and S. S. Dragomir (Nova Science Publishers, New York, 2007), Vol. 4, p. 137.

[65] S. Wissmann, A. Karlsson, E.-M. Laine, J. Piilo, and H.-P. Breuer, Phys. Rev. A 86, 062108 (2012).

[66] R. R. Puri, Physics and Astronomy Online Library (Springer, Berlin, 2001).

[67] A. Smirne and B. Vacchini, Phys. Rev. A 82, 022110 (2010).

[68] H. Krovi, O. Oreshkov, M. Ryazanov, and D. A. Lidar, Phys. Rev. A 76, 052117 (2007).

[69] J. F. Haase, A. Smirne, J. Kołodyński, R. DemkowiczDobrzański, and S. F. Huelga, New J. Phys. 20, 053009 (2018).

[70] A. Smirne, J. Kołodyński, S. F. Huelga, and R. DemkowiczDobrzański, Phys. Rev. Lett. 116, 120801 (2016).

[71] S. N. Filippov, A. N. Glinov, and L. Leppäjärvi, Lobachevskii J. Math. 41, 617 (2020).

[72] J. Teittinen, H. Lyyra, B. Sokolov, and S. Maniscalco, New J. Phys. 20, 073012 (2018).

[73] J. Liu, X.-M. Lu, and X. Wang, Phys. Rev. A 87, 042103 (2013). 